\title{
Investigation of a novel structure for 6PolSK-QPSK modulation
}

\author{
Yupeng $\mathrm{Li}^{1,2^{*}}$, Ming $\mathrm{Li}^{1,2}$, Jiawei Han ${ }^{1,2}$ and Tingting Han ${ }^{1,2}$
}

\begin{abstract}
Benefiting from the high spectrum efficiency and power efficiency, 6PoISK-QPSK (6-ary polarization-shift keying quadrature phase-shift keying) is a promising modulation format in coherent optical communication. We proposed a novel structure to generate the 6PoISK-QPSK with two dual-drive MZMs. Simulation results show that the proposed structure can generate 6PolSK-QPSK effectively and the performance is nearly the same to the traditional one.
\end{abstract}

Keywords: 6PoISK-QPSK, DDMZM, Coherent optical communication

\section{Introduction}

In pursuit of the large transmission capacity of optical fiber communication system, advanced multilevel modulation formats are attracting great interests for the high spectral efficiency (SE) [1-3]. In fact, since power efficiency (PE) is related to the transmission distance and BER, PE is another important factor to be considered besides the SE. 6PolSK-QPSK (6-ary polarization-shift keying quadrature phase-shift keying) has attracted large attention of researchers all over the world for its advantages in both SE and PE [4-9]. 6PolSK-QPSK takes advantage of six different polarizations of optical signal without decreasing the Euclidean distance. There have been several different structures of transmitter proposed; Fig. 1 shows the most typically traditional transmitter, which needs two IQ modulators for the optical modulation, one is for I-branch and the other one is for Q-branch modulation. The original data is encoded to the four threelevel streams according to the rule proposed in [4], and the final 6PolSK-QPSK is obtained by combing the two branch optical signals. Although the structure in Fig. 1 is straightforward in signal modulation, it is worth mentioning that the IQ modulator is expensive for its complicated structure and then results in the high cost of 6PolSK-QPSK transmitter.

\footnotetext{
* Correspondence: fx_lyp@163.com

${ }^{1}$ Tianjin Key laboratory of Wireless Mobile Communications and Power Transmission, Tianjin Normal University, Tianjin 300387, China

${ }^{2}$ College of Electronic and Communication Engineering, Tianjin Normal University, Tianjin 300387, China
}

In this paper, we proposed a novel structure for 6PolSK-QPSK modulation based on dual-drive MZM (DDMZM), which can reduce the complexity and cost of the transmitter. Simulation results show that the proposed structure is effective to generate the 6PolSKQPSK, and the performance is nearly the same to the traditional one.

\section{Theoretical analysis}

Figure 2 shows the structure of DDMZM; it consists of two phase modulators. The $V_{\mathrm{RF} 1}$ and $V_{\mathrm{RF} 2}$ ports are driven by the electrical signal to change the phase of optical signal. $V_{\mathrm{d} 1}$ and $V_{\mathrm{d} 2}$ ports are used to set the phase deviation between upper and lower phase modulator. Because $R F_{1}$ and $R F_{2}$ of the DDMZM can be adjusted independently, the DDMZM has high degree of freedom. In fact, a DDMZM can be used to generate any highorder modulation theoretically [10]. Based on the high degree of freedom of DDMZM, we can utilize it to generate the 6PolSK-QPSK.

According to the coding rules proposed in [4] (Fig. 3), nine bits are encoded to two consecutive symbols. The 6PolSK-QPSK modulation can be divided into two parts, that is, DP-QPSK and PS-QPSK generation. In other words, the DDMZM is used for DP-QPSK or PS-QPSK generation at a moment.

As showed in Fig. 3, the first step is encoding nine bits to two symbols. When the first bit is 0 , the subsequent bits are encoded to two DP-QPSK symbols; if the first bit is 1 , the subsequent coding method is 


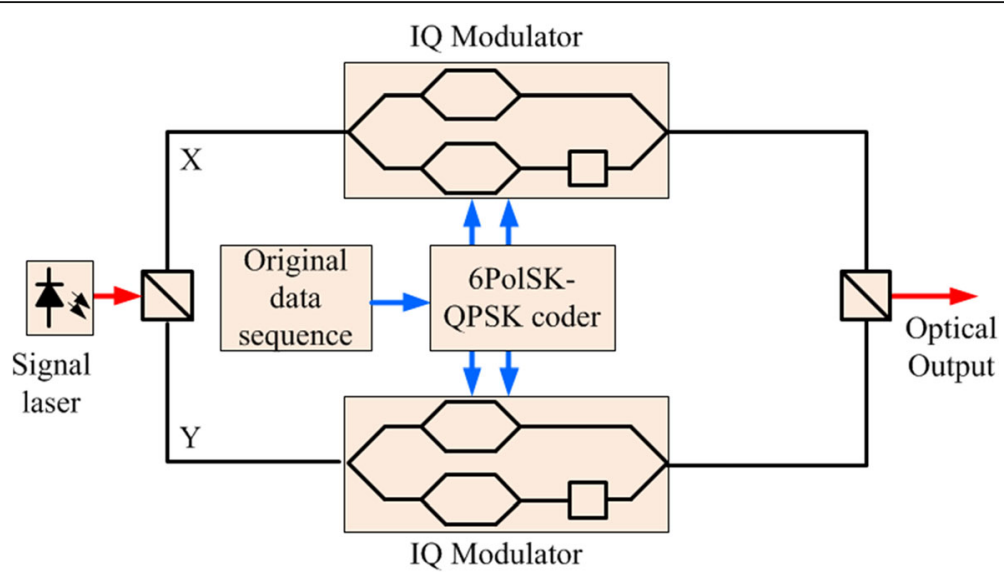

Fig. 1 The most typically traditional transmitter

decided by the second bit and, then, PS-QPSK or DP-QPSK are encoded with different sequence according to the second bit.

Figure 4 shows the I-part constellation of 6PolSKQPSK; the constellation consists of two parts. The red points represent the points for DP-QPSK, and the blue points represent the points for PS-QPSK. >For the DP-QPSK, both polarizations are QPSK modulation, and the phase in each polarization is one of the four red points; then, we obtain 16 cases. For the PSQPSK, one polarization is QPSK modulation and the other is no light, and forms the other five blue points in the constellation; the PS-QPSK has 8 cases. So we can get 24 different cases in the consecutive two symbols.

The DDMZM is suitable to generate the points in the constellation above with two independent RF signals.

Here is the transfer function of DDMZM; all the points in the constellation can be generated with different combination of the RF signals.

$$
E_{\text {out }}=\frac{E_{\text {in }}}{2}\left[\exp \left(\frac{j V_{\mathrm{RF} 1}+V_{d 1}}{V_{\pi} \pi}\right)+\exp \left(\frac{j V_{\mathrm{RF} 2}+V_{d 2}}{V_{\pi} \pi}\right)\right]
$$

where $V_{\pi}$ is the voltage to make the optical signal get $\pi$ phase shift in the phase modulator. We assume four basic phase shift for the modulation, $P=(\pi / 4,3 \pi / 4,5 \pi /$ $4,7 \pi / 4)$, the green arrows in Fig. 4 represent the basic phase shift in $P$, and all the points in the constellation can be generated with the four basic phase shift. Because the maximum phase difference in $P$ is $3 \pi / 2$, the $V_{\mathrm{pp}}$ of the driven signal is $3 V_{\pi} / 2$.

The optical signal $E_{\text {in }}$ is split into two parts $E_{1}$ and $E_{2}$, both initial phases of $E_{1}$ and $E_{2}$ are 0 , and with different $V_{\mathrm{RF}}$, the phase of $E_{1}$ and $E_{2}$ is belong to basic phase shift $P$. After combing the $E_{1}$ and $E_{2}$, the corresponding point is obtained at the output of DDMZM. For example, if the $E_{1 \_ \text {phase }}=\pi / 4$ and $E_{2 \_ \text {phase }}=3 \pi / 4$, we can get the upper red point in DP-QPSK, and when $E_{1 \_ \text {phase }}=\pi / 4$ and $E_{2 \_ \text {phase }}=\pi / 4$, we can get the upper right blue point in PS-QPSK.

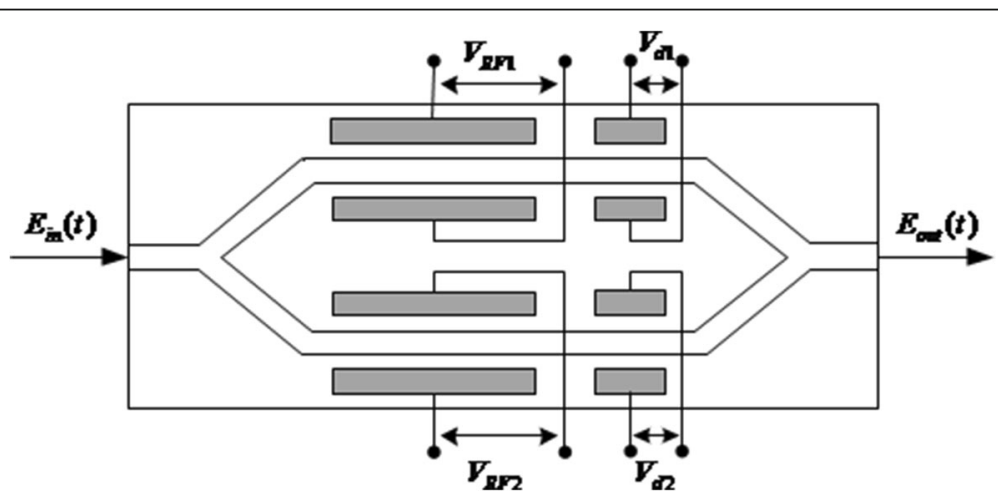

Fig. 2 The structure of DDMZM 


\section{$\begin{array}{lllllllll}b_{1} & b_{2} & b_{3} & b_{4} & b_{5} & b_{6} & b_{7} & b_{8} & b_{9}\end{array}$}

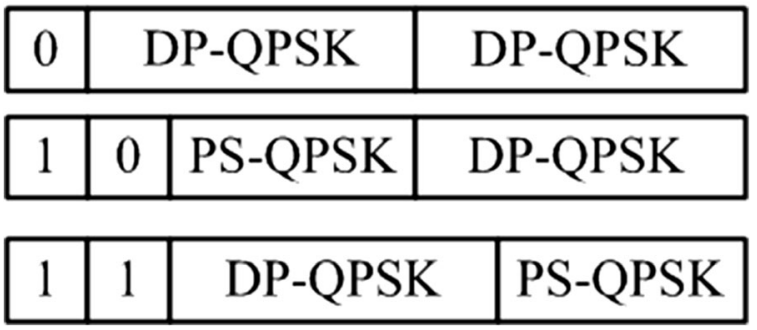

Fig. 3 Nine bits are encoded to two consecutive symbols

In order to get the four basic phase shift, the driven signals should have four different levels to form the points in DP-QPSK or PS-QPSK. With the coding rules, we can get the 6PolSK-QPSK at the output.

In addition, the DDMZM can work at different bias point, such as peak, null, and quad. For different bias point, the initial phase shift is different between upper branch and lower branch; the coding needs minor adjustment. We take null points for example, which means the two branches in DDMZM have phase difference $\pi$, so when the $V_{\mathrm{RF} 1}=V_{\pi} / 4$ and $V_{\mathrm{RF} 2}$ $=V_{\pi} / 4$, with the phase difference $\pi$, the output is the zero point in PS-QPSK.

\section{Simulation results and discussion}

We did some simulations to verify the performance of the proposed structure. Figure 5 is the simulation setup. At the transmitter side, the original data is encoded to four data streams according to the coding principle [4]; two DDMZMs are used for the I- and Q-part modulation respectively. According to the theory analysis above, the driven signals are four-level signals with $V_{\mathrm{pp}}=3 V_{\pi} / 2$; the $\mathrm{PBS}$ and $\mathrm{PBC}$ are used for split and combine the different polarization of the light signal. Both linewidths of lasers used as signal laser and local laser are $0 \mathrm{~Hz}$, so the influence of frequency deviation and phase noise can be eliminated. The wavelength of the lasers is $1550 \mathrm{~nm}$. The 6PolSK-QPSK is combined with the ASE (amplified spontaneous emission) noise to adjust the OSNR (optical signal-to-noise ratio) in the back to back simulation system. The simulation symbol rate is $10 \mathrm{GBd}$, because each 6PolSK-QPSK symbol contains 4.5 bits; the total data rate is $45 \mathrm{~Gb} / \mathrm{s}$.

Coherent detection is used to recover the signals. The local light is combined with the signal light in two $2 \times 4$ $90^{\circ}$ Hybrids and converted to electrical signals with four balanced photo-diodes (BPD), obtaining I-part and Qpart signals in both $X$ and $Y$ polarization, respectively. The four signals $R_{\mathrm{XI}}, R_{\mathrm{XQ}}, R_{\mathrm{YI}}$, and $R_{\mathrm{YQ}}$ are stored and decoded according to the rules above.

In the simulation, we assume all the parts of the system are ideal, and evaluate the performance of the proposed structure with the EVM and BER.

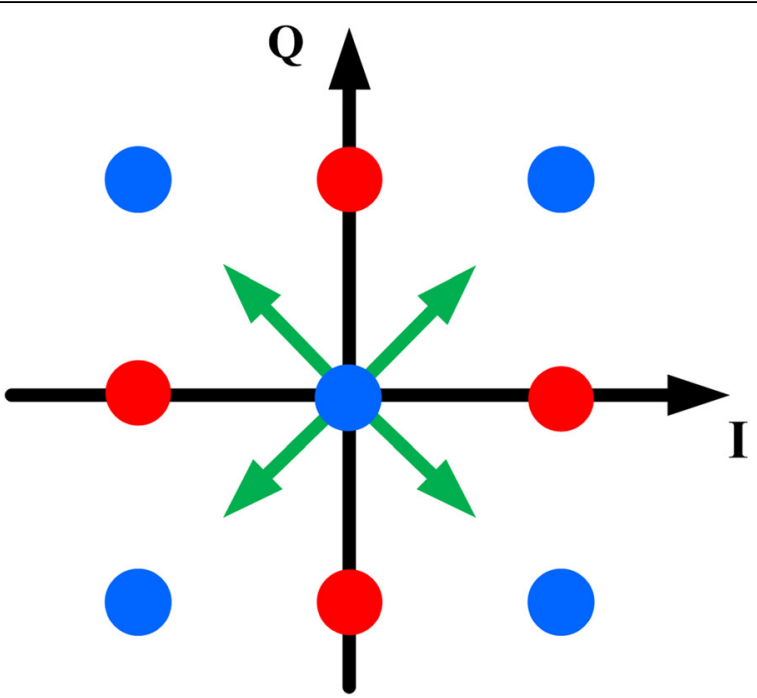

Fig. 4 The I-part constellation of 6PolSK-QPSK 

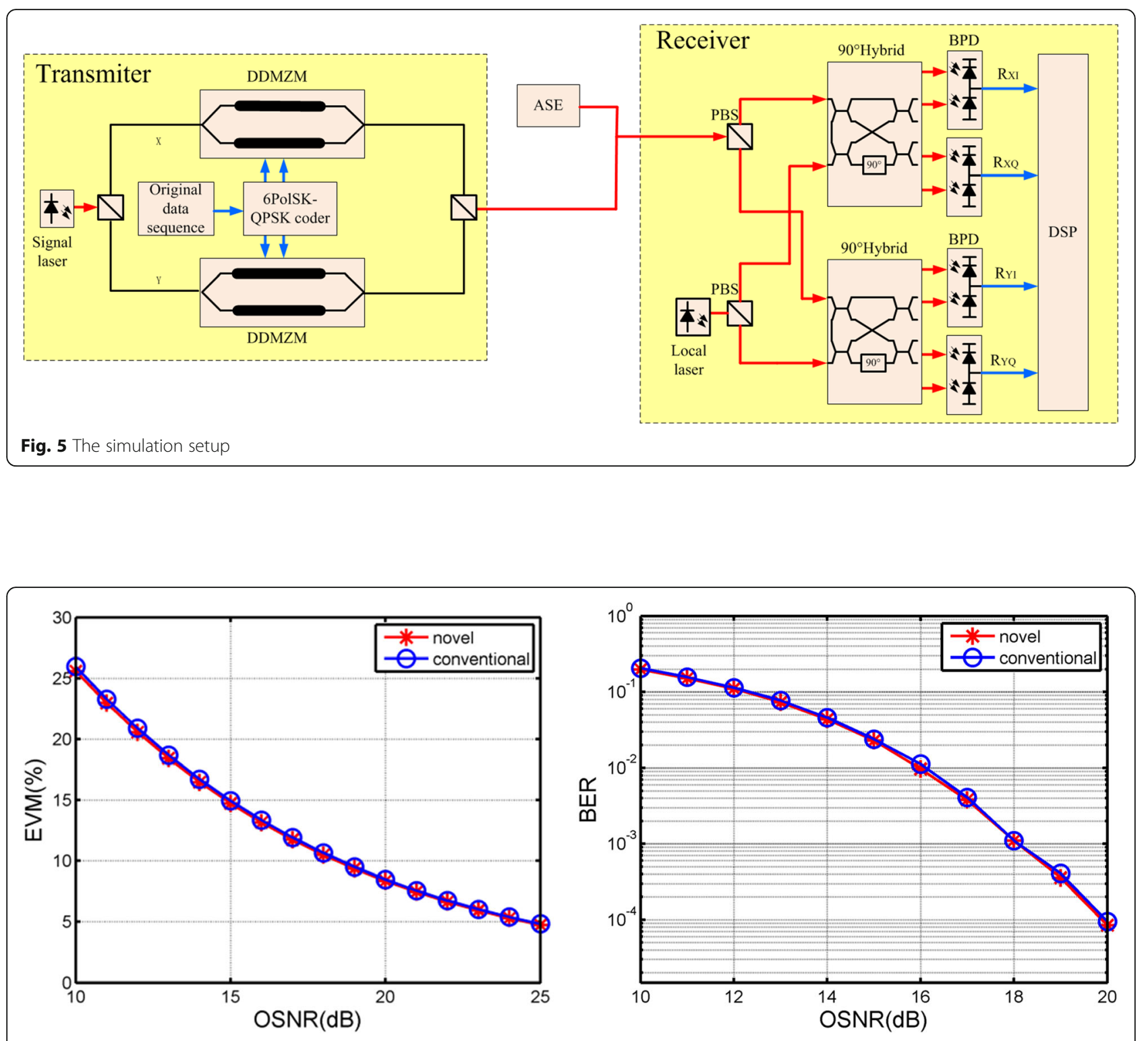

Fig. 6 The EVM and BER results with different OSNR $(0.1 \mathrm{~nm})$

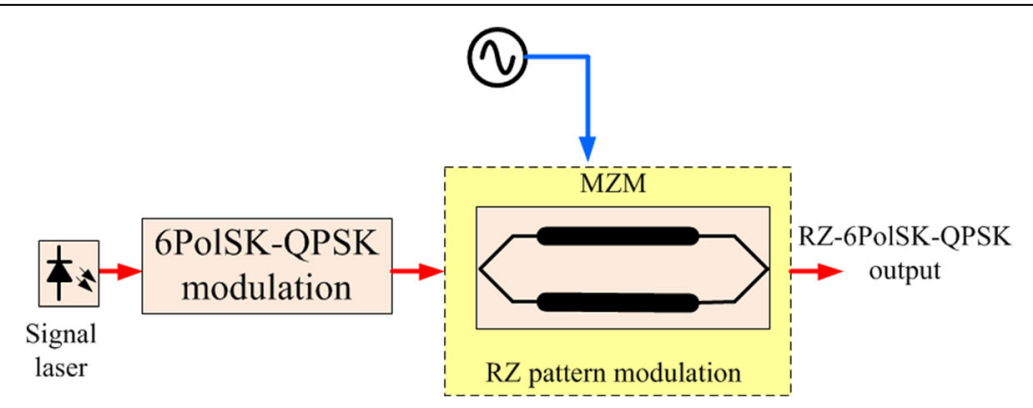

Fig. 7 The structure of RZ-6PolSK-QPSK modulation system 

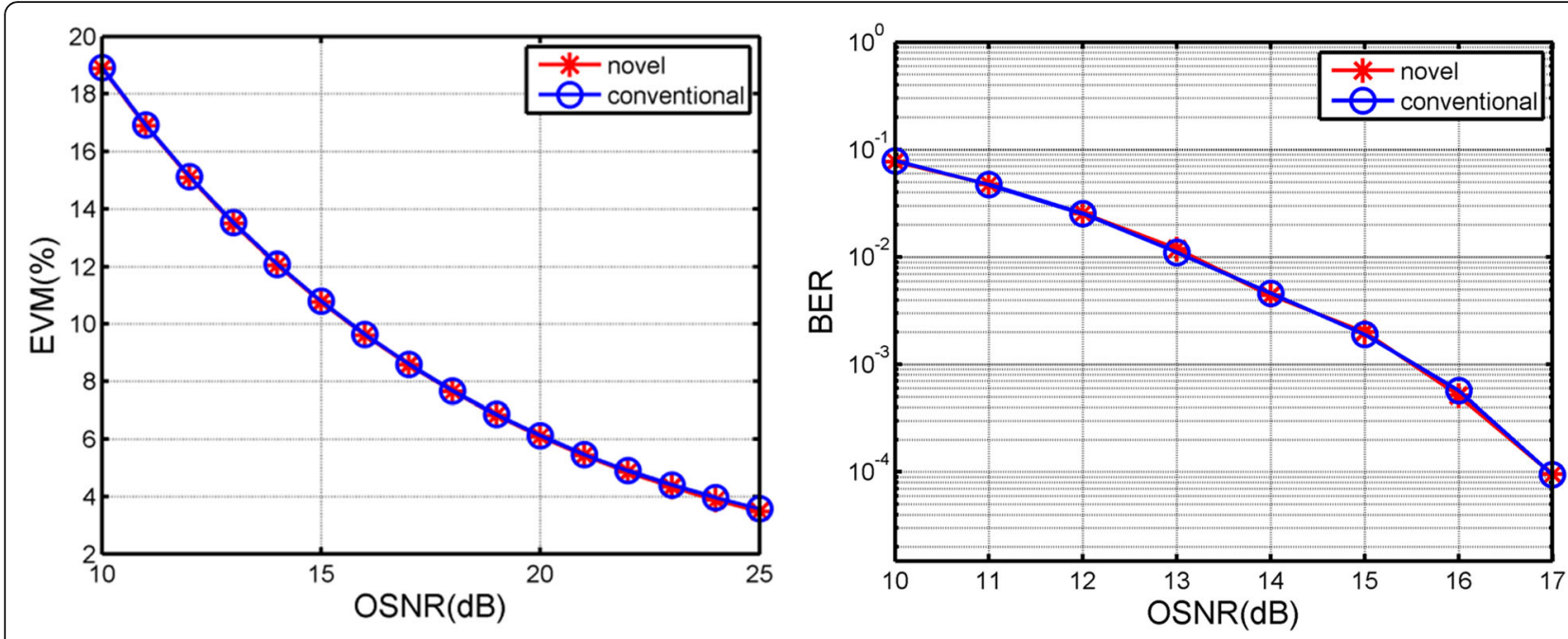

Fig. 8 The performances of RZ-6PolSK-QPSK generated by traditional and proposed structures

Figure 6 shows the EVM and BER results with different OSNR $(0.1 \mathrm{~nm})$; the results of traditional and proposed are shown together as comparisons. As shown in Fig. 6, the EVM decreased as the OSNR increased, the EVM is less than $10 \%$ when OSNR $>18 \mathrm{~dB}$, and the BER is below $10^{-3}$ when OSNR $>18 \mathrm{~dB}$. The results show the performance of the two transmitters are nearly the same; the proposed structure even has a little better performance.

For long distance transmission, the nonlinear effect of fiber will affect the system performance. Since the nonlinear effect is mainly related to the power of input optical signal, an effectively way to reduce the nonlinear effect is using the RZ pattern to reduce the average power. Figure 7 shows the structure of RZ6PolSK-QPSK modulation system; a MZM driven by sine RF signal is used for RZ pulse generation. Other parts are the same with NRZ structure.

The performances of RZ-6PolSK-QPSK generated by traditional and proposed structures are shown in Fig. 8; we get the EVM and BER results. The results show that the performance improved significantly with $R Z$ pattern. At the point of OSNR $=18 \mathrm{~dB}$, the EVM is about $8 \%$ and the BER is 0 . The RZ-6PolSKQPSK generated by the two different structures have similar performance.

\section{Conclusions}

We proposed a novel structure to generate the 6PolSK-QPSK, and a series of simulations were taken to show its performance. EVM and BER are measured to evaluate the performance of the proposed 6PolSK-QPSK system. The results show the proposed structure has similar performance with the traditional one. Furthermore, the RZ systems are researched and the results show that the RZ pattern can improve the system performance significantly. The proposed structure is an effective way to reduce the cost of 6PolSK-QPSK transmitter with the simple modulators.

\section{Funding}

This work is supported by the China Scholarship Council (201608120030), the Doctor Fund of Tianjin Normal University (52XB1505), the Doctor Fund of Tianjin Normal University (52XB1506), and National Natural Science

Foundation of China (No. 11404240).

\section{Authors' contributions}

$Y L$ is the main writer of this paper. He proposed the main idea, completed the simulation, and analyzed the result. ML and JH assisted the theory research. TH assisted the simulation. All authors read and approved the final manuscript.

\section{Competing interests}

The authors declare that they have no competing interests.

\section{Publisher's Note}

Springer Nature remains neutral with regard to jurisdictional claims in published maps and institutional affiliations.

Received: 23 January 2017 Accepted: 4 April 2017

Published online: 12 April 2017

\section{References}

1. S Beppu et al., 2048 QAM (66 Gbit/s) single-carrier coherent optical transmission over $150 \mathrm{~km}$ with a potential SE of $15.3 \mathrm{bit} / \mathrm{s} / \mathrm{Hz}$, in OFC, paper W1A. 6. (2014)

2. H Takara et al., 1.01-Pb/s (12 SDM/222 WDM/456 Gb/s) crosstalk-managed transmission with $91.4-\mathrm{b} / \mathrm{s} / \mathrm{Hz}$ aggregate spectral efficiency, in ECOC, paper Th. 3. C. 1. (2012)

3. T Omiya et al., $400 \mathrm{Gbit} / \mathrm{s}$ frequency-division-multiplexed and polarizationmultiplexed 256 QAM-OFDM transmission over $400 \mathrm{~km}$ with a spectral efficiency of $14 \mathrm{bit} / \mathrm{s} / \mathrm{Hz}$, in OFC, paper OM2A. 7. (2012)

4. E Agrell et al., Power-efficient modulation formats in coherent transmission system. J Lightwave Technol 27(22), 5115-5126 (2009)

5. M Karlsson et al., Which is the most power-efficient modulation format in optical links. Opt. Exp 17(13), 10814-10819 (2009)

6. Takahito Tanimura, et al., Nonlinear Transmission of 6PolSK-QPSK Signals using Coded Modulation and Digital Back Propagation, in OFC, paper OTu3B.3. (2013) 
7. Chen Chen, et al., Coherent Detection of a 32-Point 6PolSK-QPSK Modulation Format, in OFC, paper OTh3C. 4. (2013)

8. B Henning et al., Experimental analysis of transmission and soft decoding of optimized 4D constellations, in ACP, paper AF4C.1. (2013)

9. JK Fischer et al., Experimental investigation of 126-Gbs 6PolSK-QPSK signals, in ECOC, paper We.1.C.4. (2012)

10. H Keang-Po et al., Generation of arbitrary quadrature signals using one dual-drive modulator. J Lightwave Technol 23(2), 764-770 (2005)

\section{Submit your manuscript to a SpringerOpen ${ }^{\circ}$ journal and benefit from:}

- Convenient online submission

- Rigorous peer review

- Immediate publication on acceptance

- Open access: articles freely available online

- High visibility within the field

Retaining the copyright to your article

Submit your next manuscript at $>$ springeropen.com 